University of San Diego

Digital USD

Spring 5-31-2019

\title{
Enhancing Chronic Pain Management Motivational Interview Among Lower Back Pain Patients
}

Melissa L. Hines-Antico

University of San Diego, mlhines57@gmail.com

Follow this and additional works at: https://digital.sandiego.edu/dnp

Part of the Alternative and Complementary Medicine Commons, and the Nursing Commons

\section{Digital USD Citation}

Hines-Antico, Melissa L., "Enhancing Chronic Pain Management Motivational Interview Among Lower Back Pain Patients" (2019). Doctor of Nursing Practice Final Manuscripts. 83.

https://digital.sandiego.edu/dnp/83

This Doctor of Nursing Practice Final Manuscript is brought to you for free and open access by the Theses and Dissertations at Digital USD. It has been accepted for inclusion in Doctor of Nursing Practice Final Manuscripts by an authorized administrator of Digital USD. For more information, please contact digital@sandiego.edu. 
Enhancing Chronic Pain Management Motivational Interview Among Lower Back Pain Patients

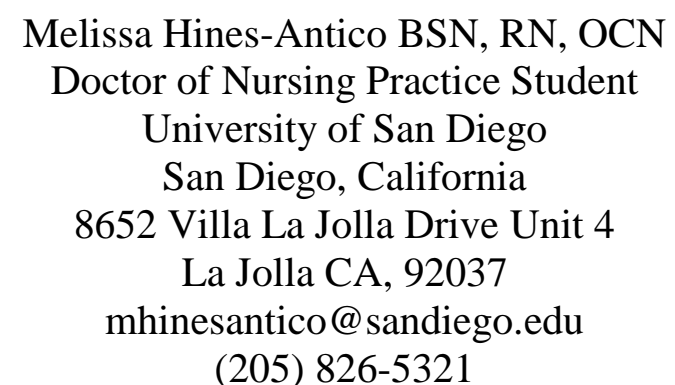

Joseph Burkard, DNSc CRNA

Associate Professor at Hahn School of Nursing

University of San Diego

San Diego, California

Gregory Polston MD

Anesthesiologist/ Pain Specialist

University of California San Diego

Medical Center

San Diego California

gpolston@ucsd.edu
Timothy Furnish MD

Anesthesiologist/Pain Specialist

University of California San Diego Medical Center

San Diego, California

tfurnish@ucsd.edu

Declarations of interest: none. 


\begin{abstract}
Purpose: Untreated chronic pain is a nationwide epidemic affect individual physically, psychologically, and financially. Low back pain is the most common subset of chronic pain. Restricted clinic time and a focus on procedural/prescriptive methods for managing pain prohibits patient education regarding self-care, and formation of a relationship with the provider. Design: Article review yielded 20 articles that supported the use of motivational interviewing for chronic pain in lower back pain patients. They were evaluated for their strength of evidence on a scale of 1-6. One article was ranked level I as a meta-analysis, and six were ranked as level II which is randomized control trials.

Methods: Implementation of monthly telephone follow-ups, guided by the 5A's framework to strengthen self-motivated behavior modifications, develop patient-centered outcomes, and outline systematic follow-up care plans. Data collection utilized standardized rating scales and questionnaires. The process proceeded for six months concluding with program evaluation.

Results: The average pain score improved from 4.6 down to $3.8(-0.8)$ almost a 1-point decrease. The average QOL score had the most dramatic increase from 4.6 to $6(+1.4)$. No improvement in pain medication usage.

Conclusion: Timely utilization of evidence-based interventions for chronic back pain can improve patient-provider interaction and promote self-care by addressing quality of life issues, decreasing patient pain scores and limiting importance of opioid medications.

Clinical Implications: Promotion of self-care behaviors encourages provider backed safety and holistic collaboration.

Keywords: Pain, Motivational Interview, Lower Back, Nurse Practitioner

Key Practice Points: The purpose of this quality improvement article is to address the gap in lack of structured follow-up in chronic pain patients. It assesses the effects of monthly telephone follow-up calls utilizing motivational interview techniques to improve pain, quality of life scores and decrease patient utilization of opioid medications. The project showed that a nurse driven follow-up resulted in lower pain scores and improved quality of life.
\end{abstract}


001

\section{Background}

002 The aim of this paper is to support the use of monthly telephone follow-up calls

003 to chronic lower back pain (LBP) patients to improve patient perceived quality of life

004 and decrease patient reported pain and opioid consumption. One of the most common,

005 and costly health conditions affecting United States (US) citizens is LBP. More U.S.

006 adults are affected by common chronic pain conditions than by heart disease, diabetes,

007 and cancer combined (Institute of Medicine, 2011). This condition affects

008 approximately $80 \%$ of people at some point in their lives, and symptom relief is needed

009 to reduce the burden of physical, psychological, and financial costs associated with LBP

010 (National Institute of Neurological Disorders and Stroke (NIH), 2014). Physically,

011 patients may be unable to work leading to disability and unemployment. In 2017 the

012 California Department of Public Health (CDPH) found that 28 out of every 100,000

013 California citizens had a work-related skeletal (back) injury and required hospitalization

014 costing upwards of \$10 billion dollars for workman's compensation (CDPH, 2017).

015 Nationally, the direct cost of treating LBP in 2014 ranged from $\$ 39$ to $\$ 78$ billion,

016 which is a conservative number considering the potential for unaccounted costs (Spine

017 Research Institute (SRI), 2014). Direct costs included traditional treatments such as

018 medication, surgery, and workman's compensation. Indirect costs were estimated to

019 total $\$ 62$ billion and considered factors such as lost productivity days and inability to fill

020 jobs vacancies (SRI, 2014). Psychologically, chronic pain is linked to anxiety and

021 depression placing patients at risk for opioid dependency (Goseling, Lin, \& Clauw,

022 2018)

023 
025 can frustrate healthcare providers resulting in passive methods of pain control like 026 opioid medication (Vong et al., 2011). This places patients at an increased risk of

027 becoming "dependent" on opioid medication and ignores alternatives such as self-

028 promoting techniques to manage the pain (Vong et al., 2011). Daily over 130 people in

029 the United States die from opioid related overdoses, and upwards of $\$ 78.5$ billion is

030 spent annually on health and social costs related to opioid abuse (National Institute on

031 Drug Abuse, 2019). In California there were over 2,000 overdose deaths reported in half

032 a years' time and in that same year $\$ 4.26$ million was spent on healthcare costs related

033 to opioid abuse (California Healthcare Foundation, 2016). Chronic conditions such as

034 LBP contribute to the opioid epidemic due to the debilitating nature of the disease. The

035 over utilization of opioids has become an epidemic in our country creating a new Health

036 People 2020 and 2030 goal to reduce the nonmedical use of opioids (2018). Opioid

037 medications are frequently inappropriately prescribed for treatment of LBP. According

038 to certain insurance reports, over half of individuals who are regular users of opioid

039 medications report LBP (Deyo, Von Korff, \& Duhrkoop, 2015). This mismanagement

040 of finances and care for LBP patients contributes to the overwhelming total cost of

041 managing chronic pain.

042 The doctoral project was implemented at the University of California at San

043 Diego Center for Pain Medicine which serves as both a treatment facility for complex

044 chronic pain conditions and provides consultation to primary care providers for

045 treatment plans. Of the patient population, $40 \%$ of patients are treated for LBP. The

046 project population included established chronic (>3 month) musculoskeletal, LBP 
047 patients actively participating in multimodal therapies. The 5A's Behavior Change

048 Model provided the framework for promotion of self-care management and use of

049 multi-modalities (Figure 1). The 5A's is a validated framework that has been utilized

050 extensively for chronic conditions requiring behavior change like obesity and smoking

051 cessation (Glasgow, Emont, \& Miller, 2006). The framework is appropriate for pain

052 management because the approach to improve self-care management. The 5A's include

053 “Ask, Assess, Advise, Assist, and Arrange". Additional recorded data includes pain

054 scales (Numeric Pain Rating Scale [NPRS]), quality of life scores (American Chronic

055 Pain Association's Quality of Life Scale [QOL] Figure 2), and number of opioid pain

056 medications (PM). Non-opioid pharmacologic interventions were considered self-

057 promoting behaviors and included topical analgesics/patches, anti-convulsant, anti-

058 seizure, muscle relaxants without benzodiazepine, and acetaminophen/nonsteroidal anti-

059 inflammatory drugs. A goal score of 5 was used for NPRS and QOL scores.

060 Data collection conducted 5 months prior to project implementation provided

061 demographics of the general population within the pain clinic. Out of 82 patients, $40 \%$

062 of randomized patients visited for LBP. Most patients are females between the ages of

06360 to 80 with a body mass index greater than 25 . The top two disturbances in patient

064 perceived quality of life included sleep and exercise. Pain and quality of life scores for 5

065 randomized LBP patients from predata collection revealed a NPRS average of 6.2,

066 average QOL 4.6, and PM average was 0.4 opioid medications (Figure 3). The random

067 pre-intervention population NPRS or QOL averages did not meet the goal score which

068 further showed areas for improvement. Only $29 \%$ the patients had a solidified follow-up 
070 regimen, and $80 \%$ of the patients needed to schedule another appointment after their

071 unplanned pain procedures.

072 The foundational question constructing the evidence for the project is: in chronic

073 lower back pain patients would a monthly telephone-follow up post-clinic visit improve

074 patient perceived quality of life and decrease patient reported pain and opioid

075 consumption. The evidence-based practice (EBP) project was formulated based on the

076 Iowa Model. The Iowa model is a validated framework that implements systematic

077 multistep processes to guide changes in healthcare (Buckwalter et al., 2017). One of the

078 most important steps is integration and sustainability of the project. A unique aspect of

079 this EBP is that the project is extended by an additional 6 months utilizing another

080 doctoral student. Our goal in having continued presence is to further embed the change

081 in practice as well as modify areas of improvement.

082 Implementation of the EBP utilized in-person and telephone interviews was

083 modeled from the 5A's behavior intervention model ([Figure 1] U.S. Preventive

084 Services Task Force, 2015). The 5 A's behavior intervention model is a validated

085 intervention that has been successfully utilized for improvement of chronic illness care

086 by promoting patient driven behavior change.

\section{Literature Review}

088 A review of literature was conducted to provide support for the project. The

089 literature review utilized the following search engines: NIH, Department of Health and

090 Human Resources, CDPH, California Health Care Foundation, CINAHL, and PubMed.

091 Keywords used for the search engines included: (Lower) back pain, motivational

092 interview, telephone base follow-up/interview/coaching, quality of life, nursing 
093 theoretical model, and pain. The keywords were combined utilizing Medical Subject

094 Headings (MeSH), and depending on the search engine utilized, yielded over 1,000

095 articles. Articles were narrowed by only including English, adult patients, published

096 after 2011, and no animals. Results of the literature review included 20 articles that

097 supported the use of motivational interviewing for chronic pain in lower back pain

098 patients. Of these articles, seven were evaluated for their strength of evidence on a scale

099 of 1-6. One article was ranked level I as a meta-analysis, and six were ranked as level II

100 which is randomized control trials. The articles assisted in structuring the intervention

101 and potential areas of analysis. Evidence-based interventions included in the study were:

102 1) Follow-up by a Nurse Practitioner (NP); 2) 5A's framework to develop a

103 questionnaire for patient driven change; 3) assess patient perceived pain, QOL, and

104 number of opioid medications prescribed; 4) promote patient centered change in

105 behavior through MI.

106 Telephone Follow-up by Nurse Practitioner

107 The project utilized a monthly telephone follow-up call to chronic pain patients

108 as a cost effective and proactive follow-up method for patients. Nurse Practitioner led

109 telephone follow-ups has been shown in the literature to be an effective method of

110 promoting behavior change and managing chronic pain. In a study by Kroenke et al.

111 (2014) found that patients who were called on a monthly basis to assess their pain and

112 how the pain interferes with the patient's activities of daily living had a 1 point decrease

113 in reported pain and reported a 30\% improvement in perceived pain. Another study

114 noted that proactive calling on behalf of the provider to the patient to discuss pain

115 resulted in a decrease of healthcare resources due to better management of external 
116 barriers (Bhimani et al., 2017). Additionally, utilizing telephone calls to deliver self-

117 management strategies in chronic pain patients delivers a multimodal approach

118 maximizing the effects of addressing pain medications, behavior change therapy, and

119 ultimately resulting in decreased pain and improved QOL (Bair et al., 2015).

120 5A's Framework for Patient Driven Change

121 Qualitative data was collected using a 5A's guided questionnaire during the

122 initial patient visit and with each monthly phone call. The questionnaire utilized the

123 5A's behavior change model by asking the following questions: (a) ask the patient their

124 readiness for utilizing self-promoting behaviors and reduction of opioids, (b) assess the

125 patients willingness to participate in self-promoting behaviors, (c) advise the patient on

126 how to utilize self-promoting behaviors, (d) assist the patient in coordinating access to

127 alternative therapies, exercise, and nutrition advise, (e) arrange for follow-up of the

128 patient with the provider within an allotted time. The American Society of

129 Anesthesiologists (ASA) in their practice guidelines strongly recommend that direct and

130 ongoing contact with the patient for their individualized treatment plan should

131 conducted on a continual basis (2010). In addition, ASA suggests that multimodal

132 interventions should be utilized for management of chronic pain. The 5A's Model

133 promotes behavior change through a step-wise delivery of validated interventions

134 (Glasgow, Emont, \& Miller, 2006). Each question in the model addresses a physical,

135 functional, psychological, or social aspect of patient care which is a recommendation of

136 the ASA for chronic pain management. Assessment of Pain, Quality of Life, and Number of Pain Medications 
139 The quantitative measurements in the project included the validated tools of

140 numeric pain scale (NPS) for patient reported pain, quality of life utilizing the American

141 Chronic Pain Association quality of life scale (QOL scale), and the recorded number of

142 opioid pain medications (PM) that the patient was currently taking. The NPS was

143 utilized because of its ability to be utilized verbally and is commonly used in the United

144 States healthcare system. Studies have shown this validated tool is the preferred method

145 for measuring chronic pain because of its comprehensibility and feasibility to be

146 completed (Hawker, Mian, Kenderska, \& French, 2011). Additionally, the NPS is the

147 tool most utilized by UC Health System. Studies show patients with LBP have a lower

148 perception of their health and well-being. Measuring QOL provides a numeric value that

149 assists in evaluating patient focused behavior change (Hidler, Whitehurst, Thomas, and

150 Foster, 2015).

151 Promote Patient Centered Behavior Change

152 Motivational interviewing (MI) has been used by multiple studies to implement

153 a biophysical approach to create meaningful interactions between the provider and

154 involves active participation by the patient to reduce pain and increase quality of life

155 (Vong et al., 2011). MI focuses attention onto the client to inspire them to improve their

156 self-belief and behaviors to achieve desired outcomes. In a study by Vong et al., patients

157 showed positive behaviors changes such as exercise, or decrease consumption of opioid

158 medication with the use of MI. Behavior adjustment is achieved through inward

159 exploration of reasons for uncertainty and resolution of that uncertainty (Chilton, Pires-

160 Yfantouda, \& Wylie, 2012). Another study utilized MI to create patent centered cgoals

161 of care with the patient, assist patients in goal achieving tasks, and develop a trusting 
162 relationship with their provider (Harman, MacRae, Vallis, \& Bassett, 2014). The goal of

163 MI is to increase QOL, decrease the patient's pain score, and develop a sense of self-

164 worth in the patient by achieving their set goals of care (Harman et al., 2014). The

165 Centers for Disease Control and Prevention (CDC) recommends that primary care

166 physicians incorporate patient motivated behavior change into their practice (2016).

167 ASA (2010) guidelines agree that cognitive behavioral therapy should be used for

168 management of chronic pain.

169

\section{Methods}

170 Study Design

171 The intervention is an evidence-based quality improvement project conducted

172 over the course of 6 months. Following completion of the project a program evaluation

173 was conducted to assess for modifications for phase II of the project. Effectiveness of

174 the intervention was calculated by comparing the average NPRS, QOL, and PM over a

175 6-month period.

176 Members of the project team included one doctoral student as the project lead,

177 and one faculty advisor who served as principal investigator. Two anesthesiologists

178 participated in patient recruitment. The project lead conducted all patient interviews,

179 recording and synthesis of data. All qualitative and quantitative data was recorded and

180 dispersed through the clinics electronic health record system.

181 IRB Approval

182 The EBP project titled "Motivation Interview in Follow-Up Telephone Calls to

183 Pain Patients to Improve Patient Outcomes" was approved by UC San Diego Human

184 Research Protections Program in October of 2017. The EBP was further approved by 
185 the IRB at University of San Diego in November of 2017. No personal patient

186 identifiers were used with any participant in the study. Patient identifiers were numbers

187 known only to the principal investigator. Prior to implementation of the project the

188 physician and the doctoral student obtained verbal consent and an information sheet was

189 provided detailing the goals of the project. There are no potential conflicts of interests or

190 financial conflicts to disclose.

191 Patient Demographics

192 Preliminary data collected indicated that LBP patients were the focus population.

193 The DNP student prior to physician clinic would review records for established, chronic

194 LBP patients participating in multimodality treatments. Clinic reviews started in July

1952018 and continued until December 2018. Outcomes assessed at each initial interaction

196 included NPRS, QOL, and PM, and subsequently would include the patient

197 questionnaire. All patients were Caucasian and ranged in age from 54 to 78 years old

198 with an average of 64 years. Four of the patients were male and one patient was female.

199 All of the male patients were overweight, and the average BMI was $26.4 \mathrm{~kg} / \mathrm{m}^{2}$ placing

200 them in the overweight category, but at a lower BMI than the national average (CDC,

201 2017). Of the patients, $40 \%$ were diagnosed with anxiety. Patient diagnoses included in

202 analysis: lower back pain (LBP), LBP with radiculopathy, lumbar facet arthropathy,

203 spinal stenosis of lumbar region, and lumbar spondylosis.

204 Project Implementation

205 The project started with a predata collection followed by phase I which was

206 implementation of the project and concluded with a program evaluation prior to start of

207 phase II of the EBP. Predata collection was performed over the course of 3 months 
208 (January 2018-March 2018). The DNP students accompanied the anesthesiologist

209 during their scheduled clinic time to assess each patient. The patients planned follow-up,

210 pain score, quality of life score, demographics, type of pain, and treatment was

211 recorded. Following the 3 month period the data was analyzed to guide the proceedings

212 for the EBP.

213 The EBP project started in July 2018 and ended in December 2018. The DNP

214 student prior to each providers clinic day would review the chart for eligible patients.

215 Eligible patients were seen in the clinic alongside the anesthesiologist. Patients were

216 given an information sheet detailing the project and verbal consent was obtained.

217 Baseline data including NPRS and QOL score was recorded during the initial visits and

218 once a month with each telephone encounter starting two weeks after the initial office

219 visit, and then on a monthly basis for six months. The questionnaire was modeled by the

220 5A's framework. Pain medications, exercise, additional treatment modalities (physical

221 therapy, acupuncture, chiropractor, and psychology), and opioid tapering were

222 documented and recorded in the patients EHR chart and sent to the physicians. At the

223 conclusion of each telephone session patient care plan and follow-up was reviewed.

224 After completion of phase I in December 2018 program evaluation was completed.

\section{Data/Results}

226 Results of phase I of the EBP NP-led telephone follow-up utilizing the 5A's

227 model for behavioral change increased patient perceived quality of and decreased

228 overall patient perceived pain score among chronic LBP patients over a 6 month period

229 (Figure 4). The average NPRS score improved from 4.6 down to $3.8(-0.8)$ almost a 1

230 point overall decrease. The average QOL score had the most dramatic increase from 4.6 
231 to $6(+1.4)$ which is a change on the scale from: "Being able to do simple chores around

232 the house and minimal activities outside of the home two hours a week" to

233 "Work/volunteer limited hours and take part in limited social activities on weekends"

234 (American Chronic Pain Association, 2019). Both pain and QOL scores met their 5-

235 score goal. PM increased by 0.2 , almost making the total average 1 pill amongst all the

236 chronic LBP patients. Of the patients, 4 out of 5 completed all 6 monthly telephone

237 follow-ups with only one patient missing 1 month due to transitioning to outside the

238 healthcare system.

239 Utilization of multimodal therapies concluded (Figure 5):

240 Psycho-behavioral: Of the 5 patients 2 of them had an underlying psychological

241 condition. Both patients were diagnosed, but not currently in treatment for anxiety.

242 Neither patient was on medication for anxiety.

243 Procedural: All of the patients had orders placed for procedures. Procedures prescribed

244 included lumbar epidural steroid injections, chemodenervation of the lumbar area, and

245 sacroiliac joint injections. All procedures were performed within UC San Diego Pain

246 clinic at a different date.

247 Physical: Of the patients, 60\% participated in a form of physical activity or was

248 instructed by the provider to perform exercises. Two of the three patients participated in

249 self-reported exercise. One of the two patients in conjunction to exercise worked with

250 water therapy which was coordinated by the clinic. The third patient was referred to and

251 participated in a physical and rehabilitation physician.

252 Pharmacological: Only one patient (20\%) was treated with a medication. This was prescribed post major surgical procedure with a solidified plan for dose reduction and 
253 stop date. Other patients were prescribed opioid medication but were not included in the

254 study because they were not prescribed by the anesthesiologist. Any opioid medication,

255 if deemed appropriate to the patient, was written as a recommendation in the provider

256 note but was deferred to the primary provider for prescription.

257

274 months. Related to the surgery there was a slight increase in pain medication

275 prescription, but pain improved as medication was limited with a planned opioid taper.

\section{Discussion}

Treating chronic LBP is a difficult condition without instant resolution. This EBP supports alternatives for managing pain. The NP-led telephone follow-up project achieved two of the goals established by multiple pain organizations by decreasing patient perceived pain and improving QOL scores. Pain scores improved by almost 1point reduction from 5 to 4; and QOL increased by an impressive 1.4 points from 4.6 to

6. Compliance was at $80 \%$ over a 6 -month period. To note, every patient who was consented for the project agreed to participate. Each telephone encounter was allotted 15 minutes, multiple times follow-up phone calls occurred over 30 minutes which was a benefit and a limitation.

The intervention proved to be meaningful, and highlighted the positive difference achieved when patients are supported and able to make self-changing behaviors. Quantitative data showed pain scores that peaked in October and then drastically declined in December. This can be attributed to an increase need for procedural interventions prior to the holiday season as outlined in patient interviews. Patients also noted an increase in stress prior to the holidays. One patient had major back surgery prior to October which led to high levels of pain, improving over 3 
276 QOL scores reflected patient appreciation of a clinic call, and feelings of individualized

277 treatment. Patients were eager to discuss efficacy of procedures and how they utilized

278 self-care such as exercise, improved sleep, alternate therapies or overall sense of

279 wellbeing.

280 UCSD hospital currently has telemedicine capabilities. It is accredited as a Clear

281 Health Quality Institute (CHQI) meaning the health system can provide consumer-to-

282 provider, provider-to-consumer, and provider-to-provider telemedicine. Reimbursement

283 for telemedicine at UCSD hospital is achieved through contracts with participating

284 clinics. Providers using telemedicine act as a consultant and as such do not prescribe

285 treatments or medications. Appointments at UCSD Pain Clinic are typically 30-minutes

286 and for some patients that is an insufficient amount of time. Telehealth is not utilized by

287 the Pain Clinic at UCSD, but continuation of the project could provide an incentive to

288 assess the ability to be reimbursed directly for telephone calls to patients.

Implications for Nursing

290 As shown by the increased perception of QOL, the 5 A's framework is a

291 valuable tool for promoting self-care behaviors in patients. The ability of the 5 A's

292 framework to be tailored to address the individualized needs of each patients promoted a

293 sense of independence. Patients were able to evaluate their own goals and define their

294 role in achieving pain relief. As a provider, the 5 A's allowed the NP to guide the

295 patient in a supportive role that fostered a symbiotic patient-provider relationship. The

296 results supported the need for multimodal approach utilizing scheduled follow-up,

297 procedures, and exercise to decrease pain. For phase II, DNP students are focusing on

298 increasing patient study size and return in clinic visits with the provider. 
299

300

301

303

\section{Limitations/Sustainability}

The major limitation associated with the project was small sample size. At the

pain clinic there are no nurses or nurse practitioners. Much of the project was collecting and analyzing predata to show a gap in care at the clinic, and how a nurse practitioner intervention is beneficial. Positive results from the EBP project support the need for NPled follow-up for LBP patients, and would be further enforced with larger sample size. Phase II of the project aims at least double the current sample size. In addition, this project was performed in a wealthy urban area that primarily serves older, Caucasian adults. Implementation and feasibility of the project could be better assessed with increased exposure to rural and minority population.

Sustainability can be achieved by a dedicated staffer. Currently, the two phases last 6 months and are performed by DNP students. However, as outlined in the cost benefit analysis it would be cost effective to hire a medical assistant to perform telephone follow-up on a continual basis. Other options include exploration into a NP presence within the clinic to provide close follow-up.

\section{Cost Benefit Analysis}

The UCSD pain clinic averages 2,000 to 3,000 new LBP patients yearly. There are 9 providers within the clinic and two participated in the nurse practitioner protocol. If $20 \%$ of patients seen by the two providers return to the clinic once of an additional visit as a result of the telephone follow-up there is a potential profit of $\$ 13,132.84$ annually. In addition, from our sample size $100 \%$ of patients received an injection to treat LBP within 6 months. Conservatively, if only $80 \%$ of new LBP receive one

21 injection twice a year there is a potential profit of $\$ 73,682.69$. If a medical assistant was 
322 hired to make telephone calls the starting salary at UCSD is $\$ 37,416.96$ there would still

323 be a profit of $\$ 50,549.80$. This project cost nothing to implement besides time and one 324 person to perform calls.

\section{Conclusion}

326 Phase I of the EBP project supported the use of a NP driven telephone follow-up

327 to support and improve outcomes amongst chronic LBP patients. One of the greatest

328 areas of potential improvement for pain management is the perception of quality of life.

329 The adaptability of the 5A's framework and the promotion of self-care in patients

330 supports a sense of self worth and independence in the patient. The utilization of

331 telephone calls showed to be a viable and cost-effective method of interaction with

332 patients that promotes healthcare access. With continuation of the project, goal is to

333 provide further insight into the importance of close follow-up with chronic pain patients

334 and provide insight into varying methods of pain management. 
Figure 1

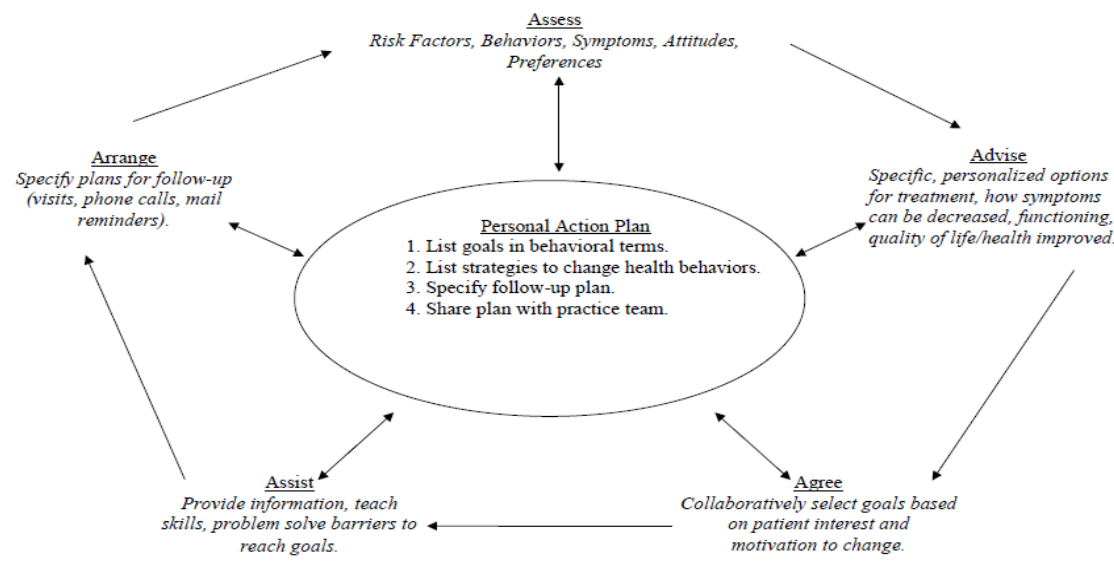

From "Self-Management Aspects of the Improving Chronic Illness Care Breakthrough Series: Implementation With Diabetes and Heart Failure Teams," by R. E. Glasgow, M. M. Funell, A. E. Bonomi, C. Davis, V. Beckham, and E. H. Wagner, 2002, Annals of Behavioral Medicine, 24, p. 83. Copyright 2002 by Springer. Adapted with permission.

Figure 1: Explanation of 5A's Framework 
Figure 2

\begin{tabular}{|c|c|}
\hline \multicolumn{2}{|c|}{$\begin{array}{l}\text { Quality Of Life Scale } \\
\text { A Measure Of Function } \\
\text { For People With Pain }\end{array}$} \\
\hline $\begin{array}{r}\mathbf{0} \\
\text { Non-functioning }\end{array}$ & $\begin{array}{l}\text { Stay in bed all day } \\
\text { Feel hopeless and helpless } \\
\text { about life }\end{array}$ \\
\hline 1 & $\begin{array}{l}\text { Stay in bed at least half the day } \\
\text { Have no contact with outside world }\end{array}$ \\
\hline 2 & $\begin{array}{l}\text { Get out of bed but don't get dressed } \\
\text { Stay at home all day }\end{array}$ \\
\hline 3 & $\begin{array}{l}\text { Get dressed in the morning } \\
\text { Minimal activities at home } \\
\text { Contact with friends via phone, email }\end{array}$ \\
\hline 4 & $\begin{array}{l}\text { Do simple chores around the house } \\
\text { Minimal activities outside of home two } \\
\text { days a week }\end{array}$ \\
\hline 5 & $\begin{array}{l}\text { Struggle but fulfill daily home } \\
\text { responsibibilities No outside activity } \\
\text { Not able to workvolunteer }\end{array}$ \\
\hline 6 & $\begin{array}{l}\text { Work/volunteer limited hours } \\
\text { Take part in limited social activities } \\
\text { on weekends }\end{array}$ \\
\hline 7 & $\begin{array}{l}\text { Workvolulunteer for a few hours daily. Can } \\
\text { be active at least five hours a day. Can } \\
\text { make plans to do simple activities } \\
\text { on weekends }\end{array}$ \\
\hline 8 & $\begin{array}{l}\text { Work/volunteer for at least six hours daily } \\
\text { Have energy to make plans for one evening } \\
\text { social activity uring the week } \\
\text { Active on weekends }\end{array}$ \\
\hline 9 & $\begin{array}{l}\text { Work/volunteer/be active eight hours daily } \\
\text { Take part in familyly life } \\
\text { Outside social activities limited }\end{array}$ \\
\hline 10 & $\begin{array}{l}\text { Go to workvolunteer each day } \\
\text { Normal daily activities each day } \\
\text { Have a social life outside of work }\end{array}$ \\
\hline
\end{tabular}

Figure 2: The American Chronic Pain Association Quality of Life Scale 
Figure 3

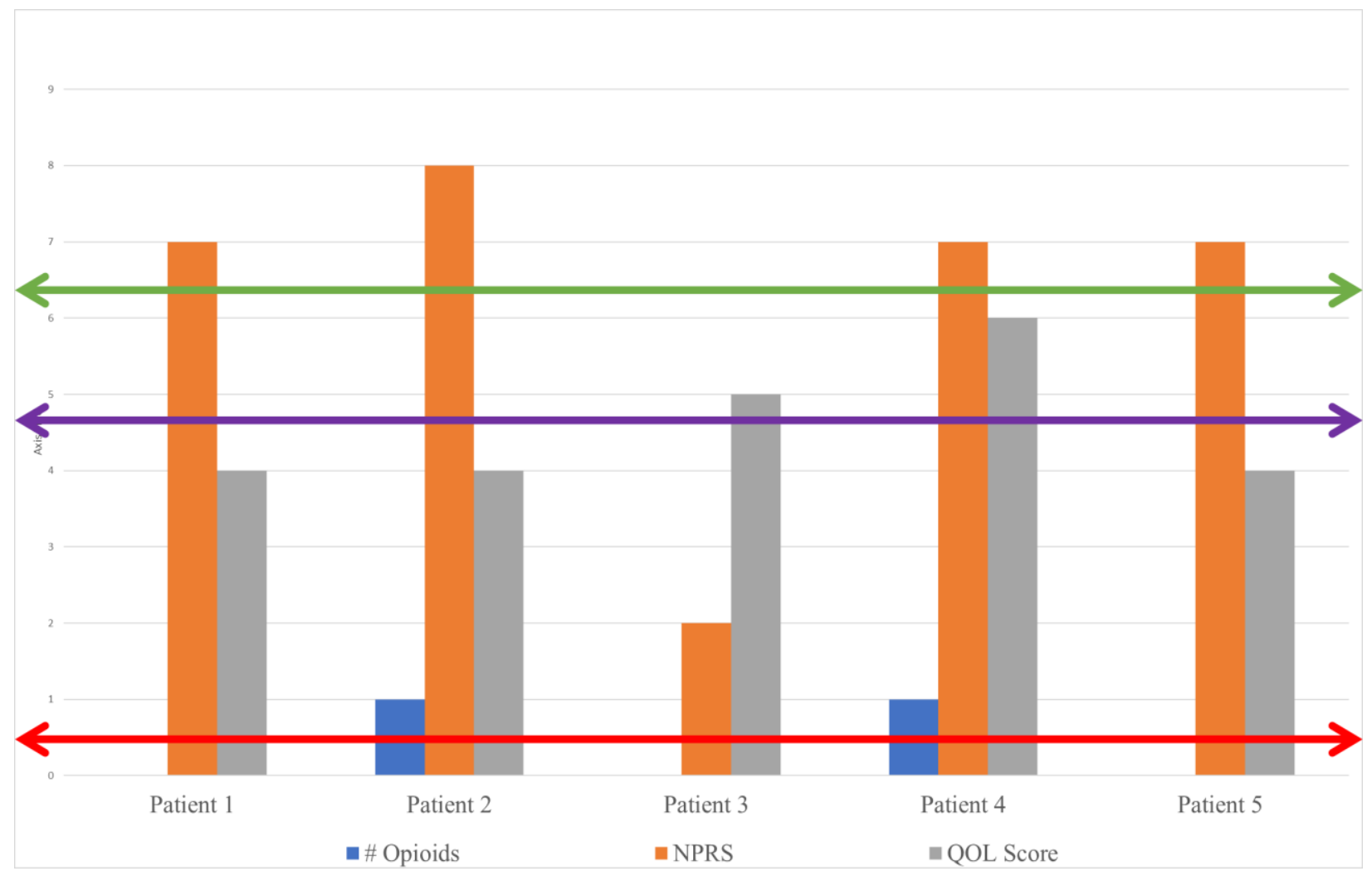

Figure 3: Preliminary baseline data number of opioid medications, quality of life, and pain score. 
Figure 4

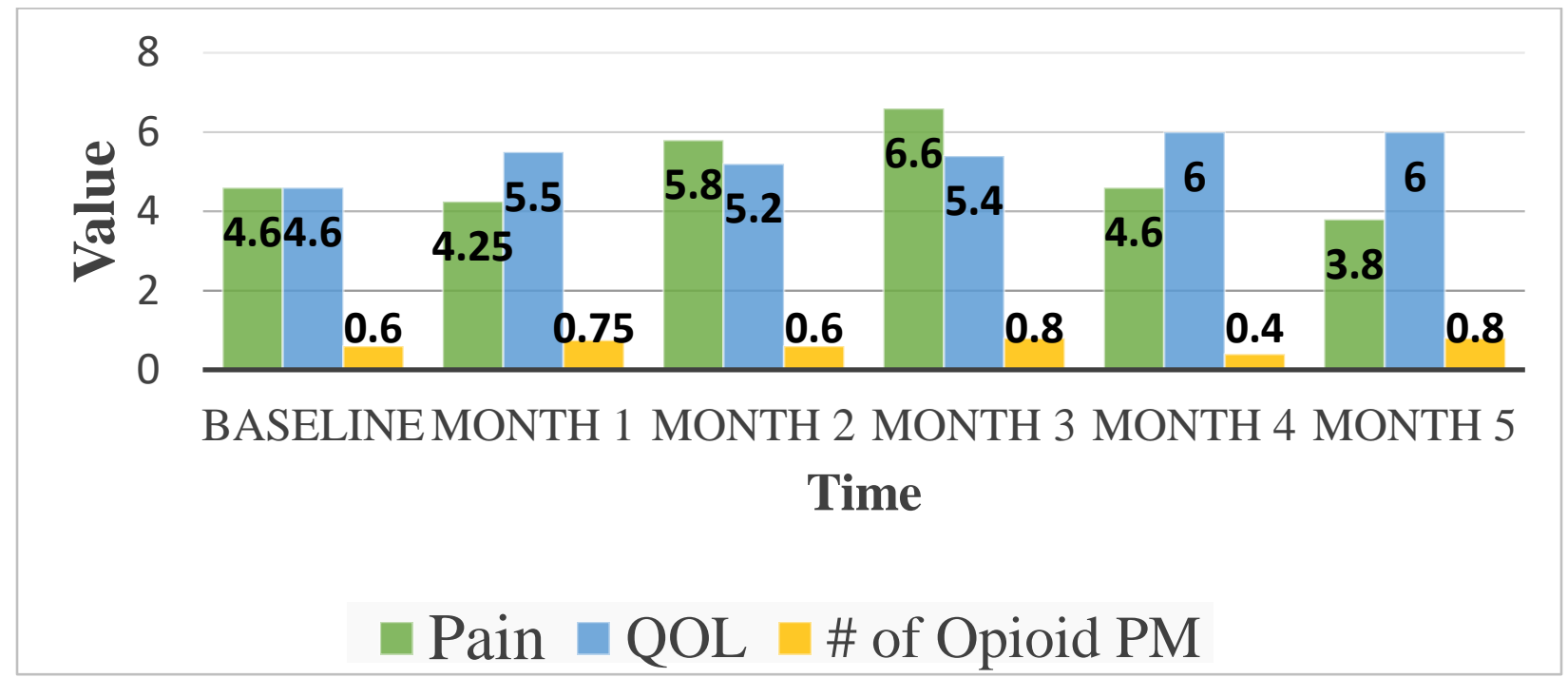

Figure 4: Comparison of the average pain scores, quality of life scores, and number of opioid pain medications over six months. 


\section{Figure 5}

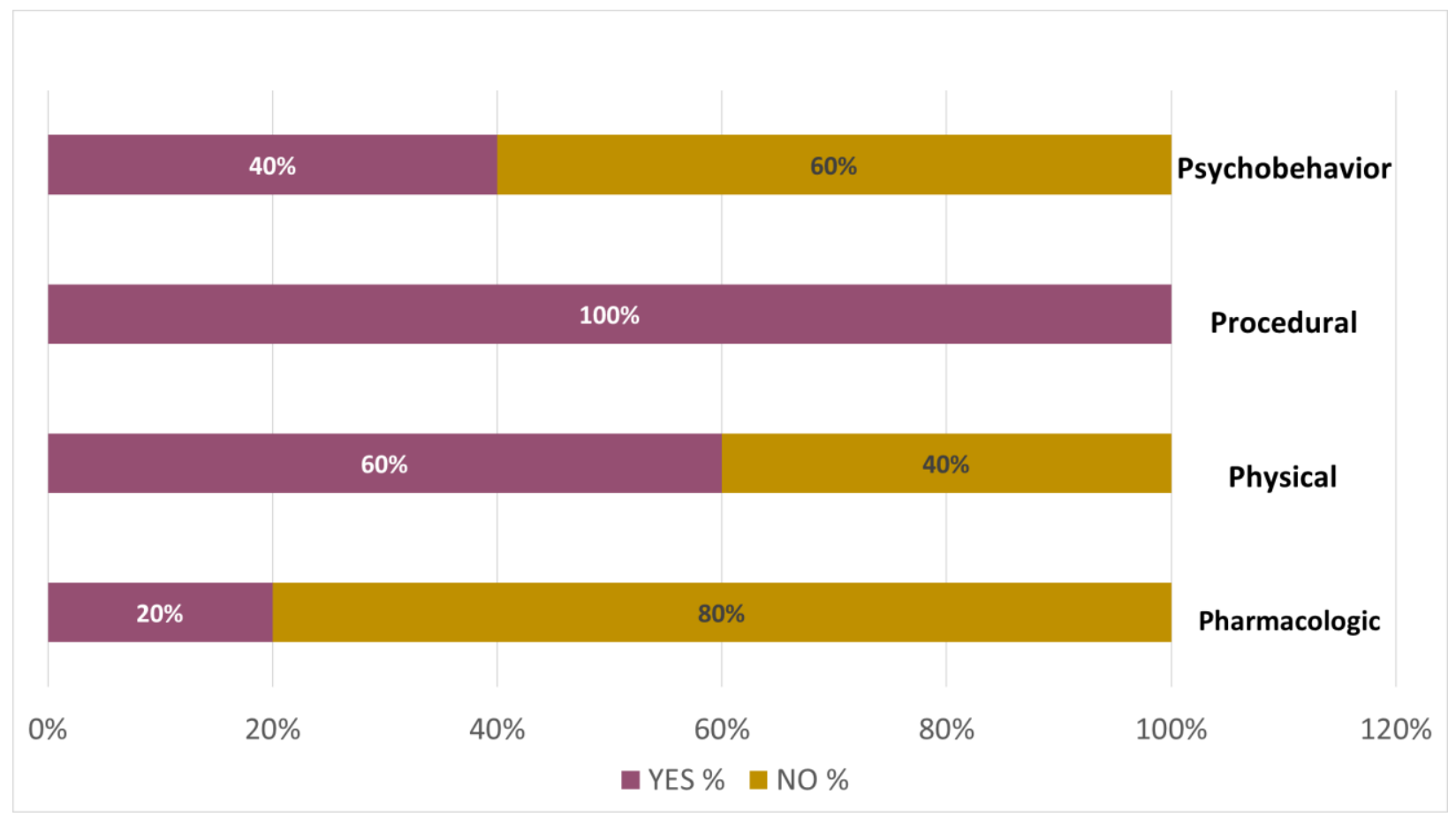

Figure 5: Utilization of multimodal therapies amongst sample population 


\section{Acknowledgements}

I would like to thank my husband, Jay Antico for his support and encouragement throughout my education and project completion. I would also like to thank my family Dennis Hines, Cheryl Hines, and Jessica Hines for inspiring me each and every day. Thank you to Valerie Gates BSN RN who is continuing this project. Lastly to all my family, friends, and teachers thank you for believing in me and supporting me throughout this journey. 


\section{References}

American Chronic Pain Association. (2003). Quality of Life Scale. Retrieved from: https://www.theacpa.org/wp-content/uploads/2017/08/Life_Scale_3.pdf

American Society of Anesthesiologists (2010). Practice guidelines for chronic pain management. Anesthesiology, 112 (4), 1-24. Retrieved from: file://C:/Users/mlhin/Downloads/practice-guidelines-for-chronic-pain-management.pdf

Bhimani, R., Cross, L., Taylor, B., Meis, L., Steven, S., Allen, K.,... Burgess, D. (2017). Taking ACTION to reduce pain: ACTION study rationale, design and protocol of a randomized trial of a proactive telephone-based coaching intervention for chronic musculoskeletal pain among African Americans. BMC Musculoskeletal Disorders, 18(15), 1-14. doi: $10.1186 / \mathrm{s} 12891-016-1363-6$

Bair, M., Ang, D., Wu, J., Outcalt, S., Sargent, C., Kempf, C., ... Kroenke, K. (2015). Evaluation of stepped care for chronic pain (ESCAPE) in veterans of the Iraq and Afghanistan conflicts: a randomized clinical trial. JAMA Internal Medicine 31(6), 470-492.

Buckwalter, K., Cullen, L. Hanrahan, K., Kleiber, C., McCarthy, A., Rakel, B., ... Tucker, S. (2017). Iowa model of evidence-based practice: Revisions and validation. Sigma Journal. doi: 10.1111/wvn.12223

California Department of Public Health. (2017). California Occupational Health Indicators: Annual measures of worker health and safety for years 2008- 2013. Retrieved from: https://www.cdph.ca.gov/Programs/CCDPHP/DEODC/OHB/CDPH\%20Document\%

California Health Care Foundation. (2016). How the opioid epidemic affects California 
Retrieved from: http://californiahealthline.org/multimedia/how-the-opioid-epidemic affects-california/

Center for Disease Control and Prevention. (2016) Contextual evidence review for the CDC guideline for prescribing opioids for chronic pain- United States. Retrieved from https://stacks.cdc.gov/view/cdc/38027

Center for Disease Control and Prevention. (2017). Healthy Weight: About Adult BMI Retrieved from https://www.cdc.gov/healthyweight/assessing/bmi/adult_bmi/index.html

Chilton, R., Pires-Yfantouda, R., \& Wylie, M. (2012). A systematic review of motivational interviewing within musculoskeletal health. Psychology, Health \& Medicine, 17 (4), 392-407. doi: 10.1080/13548506.2011.635661

Department of Health and Human Services. (2016). The opioid epidemic: By the numbers. Retrieved from: http://www.hhs.gov/sites/default/files/Factsheet-opioids-061516.pdf

Deyo, R., Von Korff, M., \& Duhrkoop, D. (2015). Opioids for low back pain. BMJ, 1-13. doi: 10.1136/bmj.g6380

Glasgow, R., Elmont, S., \& Miller, D. (2006). Assessing delivery of the five 'As' for patient centered counseling. Health Promotion International, 21(3), 245-255. doi: 10.1093/heapro/dal017

Goesling, J., Lin, L., \& Clauw, D. (2018). Psychiatry and pain management: At the intersection of chronic pain and mental health. Current Psychiatry Reports, 20 (12), 11-19. doi.org/10.1007/s11920-018-0872-4 
Harman, K., MacRae, M., Vallis, M., \& Bassett, R. (2014). Working with people to make changes: A behavioral change approach used in chronic low back pain rehabilitation. Physiotherapy Canada, 66(1), 82-90. doi: 10.3138/ptc.2012- 56BC

Hawker, G., Mian, D., Kendzerska, T., \& French, M. (2011). Measures of adult pain: Visual Analog Scale for Pain, Numeric Rating Scale for Pain, McGill Pain Questionnaire, Short Form McGill Pain Questionnaire, Chronic Pain Grade Scale, Short Form-36 Bodily Pain Scale, and Measure of Intermittent and Constant Osteoarthritis Pain. Arthritis Care \& Research, 63 (S11), 1-20. doi: 10.1002/acr.20543

Healthy People 2020. (2018) Substance Abuse. Retrieved from: https://www.healthypeople.gov/2020/topics-objectives/topic/substance-abuse

Hidler, S., Whitehurst, D., Thomas, E., \& Foster, N. (2015). Pain location matters: The impact of leg pain on health care use, work disability and quality of life in patients with low back pain. European Spine Journal, 24 (3), 444-451. doi: 10.1007s00586-014-3355-2

Institute of Medicine (IOM) Committee on Advancing Pain Research, Care, and Education. (2011). Relieving Pain in America: A Blueprint for Transforming Prevention, Care, Education, and Research. Retrieved from https://iprcc.nih.gov/sites/default/files/IOM_Pain_Report_508C.pdf

National Institute on Drug Abuse. (2019). Opioid Overdose Crisis. Retrieved from: https://www.drugabuse.gov/drugs-abuse/opioids/opioid-overdose-crisis

National Institute of Neurologic Disorders and Stroke. (2014). Lower back pain fact sheet. Retrieved from: https://www.ninds.nih.gov/Disorders/Patient-Caregiver-Education/Fact Sheets/Low-Back-Pain-Fact-Sheet 20library/AllIndicators08-13.pdf 
Spine Research Institute. (2014). Indirect costs of back pain and MSDs. Retrieved from: https://spine.osu.edu/blog/2014/09/indirect-costs-back-pain-and-msds

U.S. Preventive Services Task Force. (2015). Behavioral Counseling Interventions: An Evidence-based Approach Retrieved from https://www.uspreventiveservicestaskforce.org/Page/Name/behavioral-counseling interventions-an-evidence-based-approach

Vong, S., Cheing, G., Chan, F., So, E., \& Chan, C. (2011). Motivational enhancement therapy in addition to physical therapy improves motivational factors and treatment outcomes in people with low back pain: A randomized controlled trial. American Congress of Rehabilitation Medicine, 82, 176-183. doi: 10.1016j.apmr.2010.10.016 